P-468 DEMOGRAPHIC AND OCCUPATIONAL FACTORS ASSOCIATED TO MENTAL SUFFERING AMONG HEALTHCARE WORKERS IN BRAZIL DURING COVID-19 PANDEMIC

${ }^{1}$ Arthur Cunha, João Silvestre Silva-Junior, Cristiane Helena Gallasch. ${ }^{1}$ Federal University of Amapá, Brazil

10.1136/OEM-2021-EPI.340

Introduction COVID-19 pandemic changed healthcare routines and increased occupational stress among workers.

Objectives To analyze the factors associated with mental suffering among frontline healthcare workers (HCW).

Methods National cross-sectional study carried out in MayJune 2020 in Brazil. Participants were invited through social media to fill a questionnaire about sociodemographic, occupational, and clinical data. The Swedish Demand-Control-Support Questionnaire was applied to characterize the work psychosocial context. The World Health Organization' SelfReporting Questionnaire (SRQ-20) was used to evaluated the mental suffering. A multiple logistic regression was performed to analyze factors associated to the outcome.

Results Data were obtained from 437 workers; their average age was 38.4 years $(s d \pm 10.0)$. Most of them were from nursing team $(65.0 \%)$, female $(71.0 \%)$ and working in public services $(70.1 \%)$. Job strain was reported from $23.8 \%$ and $52.9 \%$ indicated low social support. The overall prevalence of mental suffering was $61.6 \%$ and associated factors were: : females (odds ratio - OR 1.93; 95\% confidence interval - 95\% CI $1.22-3.07$ ), age $<40$ years (OR 1.64; 95\% CI 1.07-2.52), weekly workload $\geq 60$ hours (OR 1.87; 95\% CI 1.15-3.11), job strain (OR 2.45; 95\% CI 1.41-4.40) and low social support at work (OR 3.47; 95\% CI 2.26-5.38).

Conclusion Individual characteristics and occupational aspects are associated to the suffering in this Brazilian sample of healthcare workers. Policies to improve occupational psychosocial conditions are needed in order to reduce negative mental outcomes, in special during COVID-19 pandemic.

\section{P-470 ASSOCIATION BETWEEN OCCUPATIONAL STRESS AND DEPRESSIVE SYMPTOMS AMONG HEALTHCARE WORKERS IN NORTH-EASTERN BRAZIL: A PRE- PANDEMIC SCENARIO}

${ }^{1}$ Margarete Costa Helioterio, Fernando Feijó, Fernanda De Oliveira Souza, Paloma de Sousa Pinho, Tânia Maria De Araújo. ' UFRB, Brazil

\subsection{6/OEM-2021-EPI.341}

Introduction Healthcare workers (HCW) have been reported to be at high risk for several mental health problems due to occupational stress. The outbreak of COVID-19 pandemic in early 2020 raised new concerns about the mental health of HCW workers, which may be affected by new demands and the organization of work. Studies comparing pre- and post-pandemic work environments in health settings are necessary to evaluate the effect of the pandemic on HCW's mental health. Objectives We aimed to investigate the association between occupation stress and depressive symptoms among HCW in a city in northeastern Brazil at the end of 2019, before the COVID-19 outbreak.

Methods This is a cross-sectional study with a representative sample of 355 HCW from a city in northeastern Brazil. Occupational stress, based on the demand-control model, was measured by the Job Content Questionnaire (JCQ), and depressive symptoms were evaluated by the Patient Health Questionnaire (PHQ-9). Poisson regressions were used to estimate prevalence ratios (PR) and to analyse associations of interest.

Results The prevalence of depressive symptoms (PHQ-9 $\geq 9$ ) was $22.4 \%$. Occupational stress was strongly associated with the outcome. In the crude analyses, the prevalence of depression symptoms among workers eposed to high job strain was 3.54 (95\% CI: 1.60-6.90) times that of those with low strain. After adjustment for sex, age and income, the prevalence ratio remained highly significant ( $\mathrm{PR}=3.34$; 95\%CI: 1.80-6.18).

Conclusions Occupational stress was a strong risk factor for depressive symptoms in a pre-pandemic scenario. The prevalence of depressive symptoms is expected to increase during the pandemic. Assessing occupational stress and depression during follow-up over the next few years may elucidate the impact of the pandemic on work processes and on HCW's mental health.

\section{P-471 RISK OF ENVENOMING BY POISONOUS ANIMALS IN WORKERS FROM BRAZIL (2007-2019)}

${ }^{1}$ Mariana Brito Gomes de Souza, Ana Caroline Caldas Almeida, Yukari Figueroa Mise. ${ }^{1}$ Federal University of Bahia, Brazil

\subsection{6/OEM-2021-EPI.342}

Introduction Envenomation by poisonous animals is an important public health issue in the world. The relationship between this health issue and occupation is little investigated in Brazil, and is a dimension of the accident that require more attention.

Objectives Estimate the incidence rate of poisonous animal's accidents for each occupational group and region of Brazil, between 2007-2019.

Methods Snakebite, spider and scorpion stings cases reported by Brazilian regions (2007-2019), aged 18-65 years, were collected from the Notification of Injury Information System (Sinan). The occupation identification of workers followed Brazilian Classification of Occupations (CBO). Worker population data were obtained from Brazilian Institute of Geography and Statistics (IBGE). Analyzes were conducted by Stata 16.

Results In this period, 1,523,271 accidents by poisonous animals were reported, which $49.2 \%$ were caused by scorpions, $18.4 \%$ by snakes, $17.6 \%$ by spiders and $14.8 \%$ by other etiological agents. In Brazil, the cumulative incidence for snakebite was 9.1/100,000 workers and higher in the North (36.1/ 100,000 workers). For spider stings, the national incidence was $7.9 / 100,000$ workers, with emphasis on the South (32.9/ 100,000 workers). The scorpion stings incidence was 16.1/ 100,000 workers in Brazil, and it was higher in the Northeast (24.6/100,000 workers). Occupation was reported in 480,614 $(31.6 \%)$ cases and agriculture workers were the most affected group $(51.8 \%)$. They presented the highest incidence rates among Brazil regions for all three envenomations (snakebite $=57.9 / 100,000$ workers; spider stings $=29.2 / 100,000$ workers; scorpion stings $=52.3 / 100,000$ workers).

Conclusion Rural work is considered a risk factor for envenomation by poisonous animals. The rural area is the major natural habitat for many snake species. In addition, food storage sites are attractive to prey for snakes, spiders and scorpions, such as mice and cockroaches. Knowledge about those risks 